\title{
Papillary fibroelastoma: A unique case of distant recurrence
}

\author{
Christina Popovic, MBBS, ${ }^{a}$ Matthew S. Yong, MBBS, ${ }^{a}$ Pankaj Saxena, FRACS, PhD, ${ }^{\text {a,b }}$ and \\ Sumit Yadav, FRACS, MCh, ${ }^{\mathrm{a}, \mathrm{b}}$ Townsville, Australia
}

\footnotetext{
From the ${ }^{\mathrm{a} C a r d i o t h o r a c i c ~ S u r g e r y ~ D e p a r t m e n t, ~ T h e ~ T o w n s v i l l e ~ H o s p i t a l, ~ T o w n s v i l l e, ~ Q u e e n s l a n d, ~ A u s t r a l i a ; ~ a n d ~}$ ${ }^{\mathrm{b}}$ College of Medicine and Dentistry, James Cook University, Townsville, Queensland, Australia. Disclosures: Authors have nothing to disclose with regard to commercial support.

Received for publication Aug 16, 2018; revisions received Sept 28, 2018; accepted for publication Oct 5, 2018; available ahead of print Nov 14, 2018

Address for reprints: Christina Popovic, MBBS, Cardiothoracic Surgery Department, The Townsville Hospital, 100 Angus Smith Dr, Douglas 4814, Townsville, Queensland, Australia (E-mail: christina.popovic@ health. qld.gov.au).

J Thorac Cardiovasc Surg 2019;157:e125-7

$0022-5223 / \$ 36.00$

Copyright $(C) 2018$ by The American Association for Thoracic Surgery

https://doi.org/10.1016/j.jtcvs.2018.10.035
}

Primary cardiac tumors are rare, with an incidence of $0.001 \%$ to $0.28 \%$ in autopsy series. ${ }^{1}$ The third most common primary tumor, although likely underdiagnosed, is the cardiac papillary fibroelastoma $(\mathrm{CPF}) .{ }^{2}$ These tumors are benign, do not exhibit local infiltration on histopathologic examination, and carry a low likelihood of recurrence. ${ }^{3}$ After excision, recurrence is rare, with a total of 4 reported cases of recurrence. Tamin and colleagues ${ }^{2}$ reported 3 cases, occurring at 1, 5, and 6 years after initial surgery, that they described as at or near the previous surgical excision site. Kammerer and associates ${ }^{3}$ described a case of mitral valve recurrence after 3 years but hypothesized that incomplete resection in attempt to preserve the valve was the likely underlying mechanism. We report a unique case of recurrent CPF after previous resection, occurring at multiple alternate sites throughout the left ventricle (LV).

\section{CLINICAL SCENARIO}

Our 56-year-old female patient had aortic and left ventricular CPF initially diagnosed in 2009 after presentation to the hospital with multiple transient ischemic attacks. These attacks were subsequently diagnosed as emboli from mobile intracardiac masses. The patient underwent excision of masses and aortic valve replacement (23-mm Biocor valve; St Jude Medical, St Paul, Minn), because the leaflets could not be preserved after excision of the mass.

Intraoperatively, 3 lesions were attached to the noncoronary and right coronary cusps and the undersurface of the left coronary cusp. Three lesions were removed from the base of the septum, with the largest $1 \mathrm{~cm}$ at the base of the anterolateral papillary muscle. There were no pathologic processes seen elsewhere on intraoperative transesophageal echocardiography or close macroscopic

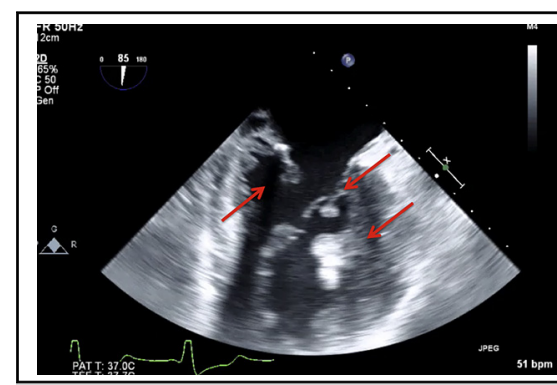

Echocardiogram of first reported fibroelastoma recurrence distant to previous resection.

\section{Central Message}

Cardiac papillary fibroelastoma recurrence is exceedingly rare but has been reported at previous resection locations. We present the first reported recurrence of distant from past surgical sites.

See Commentary on page e129.

inspection into the LV outflow tract through the excised aortic valve, within the limitations of visible pathology. The patient was discharged home after an uneventful recovery and was followed up by her local physician, with no recurrent embolic events and a normal echocardiogram. Unfortunately, on follow-up surveillance as early as 2 years postoperatively, new and recurrent mobile masses were noted. The patient received anticoagulation, and ongoing close surveillance was performed annually until degeneration of her bioprosthetic valve occurred.

By 2018, the patient had shortness of breath on exertion (New York Heart Association class III), and an echocardiogram demonstrated severe prosthetic aortic valve stenosis with recurrence of multifocal, mobile LV masses (Figure 1, A). After resternotomy, institution of cardiopulmonary bypass and standard cardioplegia, the bioprosthetic valve was excised and the LV examined endoscopically. There were numerous papillary tumors with frondlike extensions macroscopically (Figure 2,A); the largest was $9 \mathrm{~mm}$. These were attached to the LV free wall, papillary muscles, and chordae tendineae. The tumors were identified and removed with biopsy forceps, and there was no macroscopic evidence of residual tumor at completion. The aortic valve was then replaced (21-mm Trifecta, St Jude Medical). Histopathology confirmed CPFs (Figure 2,C), and previous 


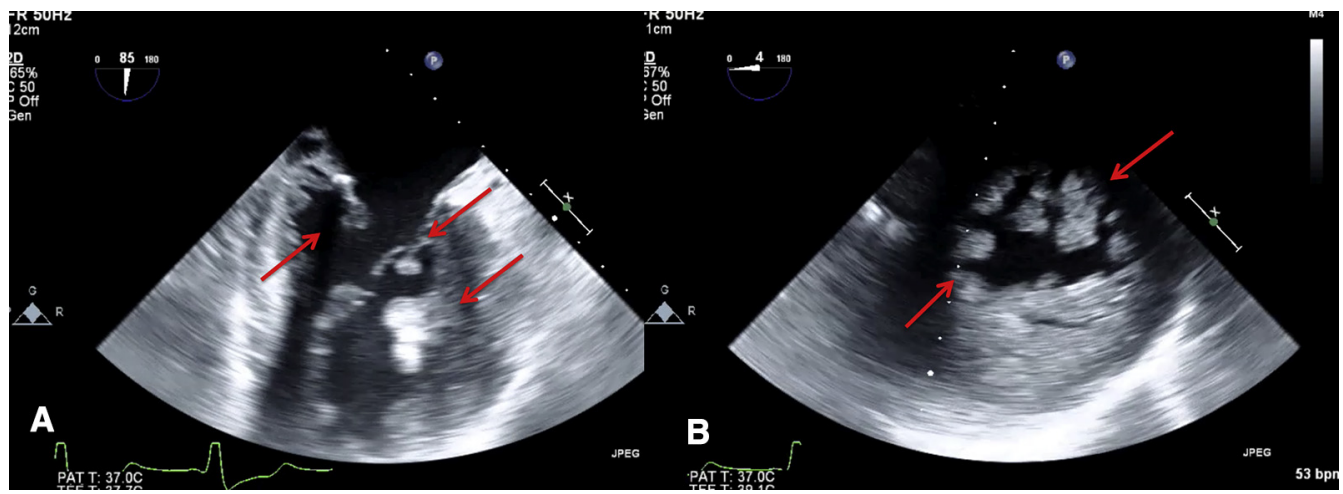

FIGURE 1. Transesophageal echocardiographic longitudinal view (A) and cross-sectional view (B) of the left ventricle with multiple papillary fibroelastomas (red arrows).

pathologic analysis was reviewed and compared to ensure that an alternate diagnosis was not missed. The patient made an uneventful recovery. Repeat echocardiography demonstrated no further recurrence of the tumors, and she remained free of symptoms at six month follow-up.

\section{DISCUSSION}

CPFs are most commonly found in the LV and then the aortic and mitral valves, respectively. ${ }^{1}$ Multiple sites are uncommon. ${ }^{4,5}$ Their clinical presentation is most commonly the result of embolic phenomena, with a transient ischemic attack, embolic stroke, peripheral or coronary emboli causing a myocardial infarction, angina, malignant arrhythmias, or sudden death. Diagnosis can be incidental in symptom-free patients.

Surgery is indicated for patients who have symptoms or are undergoing concomitant cardiac surgery. In patients without symptoms, however, indications are less well defined. The most important factor to consider for surgical intervention is the mobility of the tumor, because this is the single predictor of mortality or nonfatal embolization. ${ }^{3}$ There are no size criteria for excision; however, smaller CPFs $(4-8.5 \mathrm{~mm})$ were found to cause more neurologic events in one retrospective study. ${ }^{3}$ Outcomes after surgery are generally excellent, although follow-up is warranted because recurrence is possible. ${ }^{2}$

This case report describes recurrence of the CPF within 2 years of initial excision. Interestingly, recurrence occurred at multiple alternative sites distant from the original surgery. This has not been previously described. Tamin and colleagues $^{2}$ reported a recurrence rate of $1.6 \%$ and suggest endocardial trauma from surgery or radiation or incomplete resection as potential etiologic factors. The cause of the recurrence in the presented case is unclear. It may be related

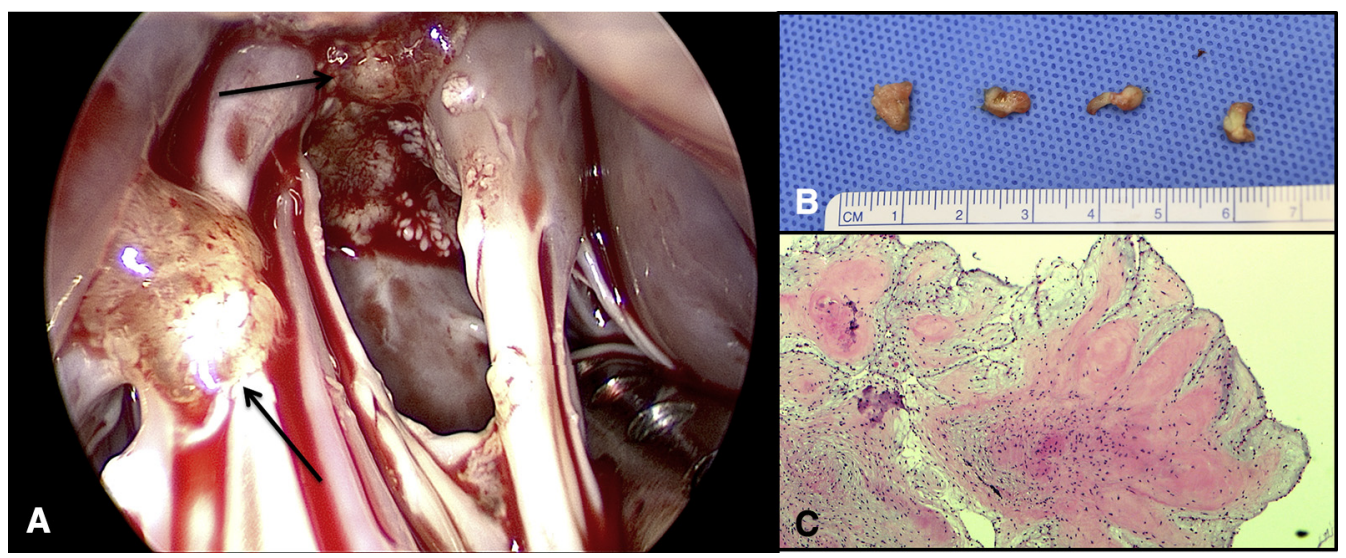

FIGURE 2. A, Intraoperative photograph of papillary fibroelastomas involving the left ventricular wall, papillary muscles, and chordae tendineae, with gelatinous appearance and frondlike projections. Arrows indicate the multiple papillary fibroelastomas. B, A representative picture of resected intraoperative specimens and their dimensions. C, Histopathologic specimen of papillary fibroelastoma with hematoxylin and eosin stain demonstrating characteristic elastic fiber arrangement in whorls surrounded by hyaline stroma and a single layer of endothelial cells. The pedunculated, highly papillary appearance can be appreciated in this section. 
to an underlying genetic predisposition, although there is no described test to confirm this. Alternatively, the underlying pathogenesis may be the result of a viral etiology, as has been previously hypothesized. We are not aware of any proposed regimens of adjuvant therapy for recurrent CPF.

\section{CONCLUSIONS}

Recurrence of CPFs after surgical resection is rare. We describe a unique case where recurrence occurred at sites distant from the original site of excision. Future biological and genetic studies are required to better understand the mechanisms of CPF pathology.

\section{References}

1. Baikoussis NG, Dedeilias P, Argiriou M, Argiriou O, Vourlakou C, Prapa E, et al Cardiac papillary fibroelastoma: when, how, why? Ann Card Anaesth. 2016;19: $162-5$.

2. Tamin SS, Maleszewski JJ, Scott CH, Khan SK, Edwards WD, Bruce CJ, et al Prognostic and bioepigemiologic implications of papillary fibroelastomas. J Am Coll Cardiol. 2015;65:2420-9.

3. Kammerer I, Besser R, Al-Azani M, Frank C, Bohrer MH, Sack FU. Fibroelastoma recurrence in left ventricle: rarity of primary cardiac tumor. Surg $J(N Y)$. 2015;1:e35-7.

4. Touati GD, Carmi D, Sevestre H, Poulain H. Multiple aortic valve papillary fibroelastoma: do not miss the other one. Eur J Cardio-Thoracic Surg. 2002;21:596-7.

5. Sato M, Nagaya K, Hatakeyama M, Komatsu T. Multiple papillary fibroelastoma: report of a case and implications for management. Gen Thorac Cardiovasc Surg. $2014 ; 62: 122-4$ 